\title{
Prevalence of serologic reactivity against four strains of mouse mammary tumour virus among US women with breast cancer
}

\author{
J Goedert ${ }^{*, 1}$, CS Rabkin' and SR Ross ${ }^{2}$ \\ 'Viral Epidemiology Branch, Division of Cancer Epidemiology and Genetics, National Cancer Institute, Rockville, MD 20892, USA; ${ }^{2}$ Department of \\ Microbiology/Cancer Center, University of Pennsylvania School of Medicine, Philadelphia, PA 19104, USA
}

\begin{abstract}
Mouse mammary tumour virus (MMTV) causes breast cancer in mice, and MMTV-specific antibodies develop to high titers among mice infected as adults. Whether MMTV or a related virus infects humans is uncertain, because MMTV DNA sequences have been detected inconsistently and because serologic methods have varied widely. The current study used immunoblot and immunoprecipitation with four strains of MMTV (RIII, FM, C3H, and LA) to detect specific antibodies in 92 sera from US women with breast cancer and in masked dilutions of monoclonal hybridoma and hyperimmunised goat positive-control reagents. In these positive controls, MMTV antibodies of the expected molecular weights were detected at high titer ( 1 : I 00 in the monoclonal reagent, I : 10000 in the hyperimmunised goat serum). Nearly $30 \%$ of the sera from women with breast cancer had at least one faint band on an immunoblot, but none of these matched the molecular weight of bands revealed by probing the same blot strips with the goat serum. The goat serum readily immunoprecipitated MMTV antigens from all four strains of MMTV, but MMTV antigens were not immunoprecipitated by any of the six breast cancer sera that had four or more nonspecific immunoblot bands. Thus, among women with breast cancer, we found no MMTV-specific antibodies. The upper 95\% confidence limit implies that MMTV seroprevalence among breast cancer patients does not exceed 3\%.
\end{abstract}

British Journal of Cancer (2006) 94, 548-55।. doi:I0.I038/sj.bjc.6602977 www.bjcancer.com

Published online 3I January 2006

(C) 2006 Cancer Research UK

Keywords: breast cancer; mouse mammary tumour virus (MMTV); seroprevalence; Western immunoblot; immunoprecipitation

\begin{abstract}
The mouse mammary tumour virus (MMTV), a betaretrovirus, causes breast cancer in mice. During the past 10 years, molecular evidence of MMTV in human breast cancer tissue has been reported by a few laboratories using polymerase chain reaction (PCR) techniques (summarised in Holland and Pogo (2004)). These findings are controversial, because other laboratories have been unable to replicate detection of MMTV and because the DNA amplified by PCR in some laboratories has had homology with nonviral sequences in the human genome (summarised in Mant et al (2004)). Serologic studies to identify MMTV antibodies complement these PCR-based molecular studies of breast cancer tissue. During the late 1970s and early 1980s, detection of serum antibodies against MMTV-infected cells or proteins from these cells among women with breast cancer provided support for the possibility of a human homologue of MMTV. There was, however, substantial heterogeneity in methods, in antigens recognised by the sera, and in seroprevalence associations. The results and limitations, particularly with respect to specificity, of these early studies were reviewed by Dion et al (1987). By Western immunoblot with disrupted, purified milk-borne MMTV of the RIII strain, Dion and co-workers found no antibodies against
\end{abstract}

*Correspondence: Professor JJ Goedert, Viral Epidemiology Branch, National Cancer Institute, 6120 Executive Boulevard, Room 8012, Rockville MD 20892, USA; E-mail: goedertj@mail.nih.gov

Received 27 June 2005; revised 26 October 2005; accepted 5 January 2006; published online 31 January 2006
MMTV viral antigens in 1:5 dilutions of sera from 30 breast cancer patients or 30 control patients (Dion et al, 1987). In $1: 8$ dilutions of sera in enzyme immunoassays, Dion et al found modestly higher reactivity against column-purified p18 from MMTV but not against four other MMTV column-purified proteins or glycoproteins in breast cancer patients compared to controls (Dion et al, 1987). Among 300 Czech subjects (90 healthy controls, 60 with breast cancer, and 150 with other malignancies or serious diseases) whose sera, diluted $1: 100$, were tested by immunoblot heavily loaded with proteins and glycoproteins from the GR/N strain of MMTV, Kovarik et al found frequent reactivity against a $42 \mathrm{kDa}$ cellular contaminant of the virus, but few with reactivity against viral antigens and no differences between cases and controls (Kovarik et al, 1989). All of the previously published studies used only a single strain of MMTV. We, therefore, sought to estimate the MMTV seroprevalence among US women with breast cancer by using a wider variety of MMTV strains. We used both immunoblotting and immunoprecipitation to maximise specificity of the anti-MMTV reactivity.

\section{MATERIALS AND METHODS}

\section{Patients and controls}

Between 1979 and 1988, the Immunodiagnosis Serum Bank collected, separated, and stored frozen sera from patients at the Mayo Clinic who had common malignancies (Dimagno et al, 
1989). To estimate MMTV seroprevalence, sera from 92 women with breast cancer in this bank were selected, based on total number of available aliquots and availability of at least one $0.25 \mathrm{ml}$ aliquot. Data on age at blood draw, histologic diagnosis, tumour stage, cancer treatment history, and smoking status were available. Identifying data had been removed. Exemption from Institutional Review Board review was obtained from the NIH Office of Human Subjects Research. Monoclonal $\alpha$-gp52 hybridoma supernatant BL6 $5 \mathrm{D}$, as well as caprine sera from goats hyperimmunised with MMTV ( $\alpha$-MMTV) or with gp52 (polyclonal $\alpha$-gp52), served as positive control reagents (Dzuris et al, 1999; Purdy et al, 2003). In a central repository, three aliquots of each of these reagents (neat, $1: 10$ and $1: 100$ dilutions in defibrinated plasma (Basematrix, BBIBiotech Inc., Frederick, MD, USA)) were put into vials identical to the human sera. The nine positive controls were interspersed among the 92 human sera and could not be identified by the laboratory. To assess the integrity of the sera, they were tested for rubella antibodies with a commercial agglutination test (BD Biosciences Inc.).

\section{Detection of MMTV-specific antibodies}

Immunoblot analysis was performed as described previously (Selmi et al, 2004). Briefly, total protein was extracted from virions purified by pelleting through sucrose cushions, as previously described (Le Bon et al, 1999). The virions were purified from mammary tumours of $\mathrm{C} 3 \mathrm{H} / \mathrm{HeN}$ mice infected with MMTV (FM) or MMTV (LA), milk from RIII mice (MMTV (RIII); a gift from Akhil Vaidya), or supernatants from the cultured mammary tumour cell line Mm5MT (MMTV (C3H)). Virions were disrupted in lysis buffer containing $25 \mathrm{~mm}$ Tris- $\mathrm{HCl}(\mathrm{pH} 8.0)$, $150 \mathrm{~mm} \mathrm{NaCl}, 2 \mathrm{~mm}$ EDTA, $0.1 \%$ sodium dodecyl sulfate, $0.5 \%$ Nonidet P-40, and $0.5 \%$ deoxycholate. The proteins were separated by sodium dodecyl sulfate-10\% polyacrylamide gel electrophoresis (SDS-PAGE) and transferred to nitrocellulose. As the virus preparations varied in purity, we normalised the amounts of virus electrophoresed in each lane by initially running different dilutions of the virus preparations and probing the filters with goat anti-MMTV serum, as described below.

Sera were kept frozen at or below $-70^{\circ} \mathrm{C}$ until testing. Each masked serum specimen was diluted $1: 100$ in phosphate-buffered saline (PBS) containing $0.1 \%$ Tween-20 (PBS-Tween) and 5\% nonfat dried milk, incubated for $5 \mathrm{~h}$ with the blots and then washed extensively with PBS-Tween. As the primary sera were masked, we used a mixture of horseradish peroxidase (HRP)-conjugated donkey anti-human (1:8000; Jackson Immunoresearch Inc., West Grove, PA), rabbit anti-goat (1:5000; Sigma Inc., St. Louis, MO) and sheep anti-mouse (1:5000; Amersham Inc., Piscataway, NJ) secondary antibodies to detect bound primary antibodies. This mixture was added to each blot in PBS-Tween-milk, incubated for $30 \mathrm{~min}$ and again washed extensively with PBS-Tween. To ensure that the mixture of secondary antibodies was species-specific, we also blindly tested 22 of the samples with the anti-human secondary antibody alone and found that the same pattern of bands seen with the mixture, except for two samples that were masked, diluted goat serum. These latter two samples showed no bands on the virus blots probed with the human secondary antibody alone but showed the typical MMTV pattern when the mixture of secondary antibodies was used (not shown). The blots were developed with ECL Enhanced Western Blotting Detection Reagent, according to the manufacturer's instructions (Amersham Inc.) To characterise whether antibodies revealed in the immunoblots had similar molecular weight to those in caprine sera, blot strips that demonstrated antibody bands were reprobed with polyclonal goat anti-MMTV antisera $(\alpha$-MMTV, $1: 3000)$ followed by the HRP-conjugated rabbit anti-goat secondary antibody. Photographs of the blots with human and $\alpha$-MMTV sera were aligned and overlaid for visual comparison. Side-by-side compar- isons are provided. To maximise detection, the human blots were exposed for up to $5 \mathrm{~min}$.

For the immunoprecipitation experiment, approximately $50 \mu \mathrm{g}$ of purified protein from each of the four MMTV strains in $200 \mu \mathrm{l}$ final volume of RIPA buffer $(25 \mathrm{~mm}$ Tris, pH 7.8, $150 \mathrm{~mm} \mathrm{NaCl}$, 2 mM EDTA, $0.5 \%$ NP40, $0.5 \%$ deoxycholate, $0.1 \%$ sodium dodecyl sulphate, $1 \mathrm{~mm}$ PMSF, $5 \mu \mathrm{g} \mathrm{ml}^{-1}$ leupeptin, $5 \mu \mathrm{g} \mathrm{ml}^{-1}$ aprotinin, $2 \mu \mathrm{g} \mathrm{ml}^{-1}$ pepstatin A) were added to $1 \mu \mathrm{l}$ of human serum, or to $1 \mu \mathrm{l}$ of a $1: 10$ dilution of the polyclonal goat anti-MMTV serum followed by $50 \mu$ l of Protein G sepharose (Invitrogen Inc., Carlsbad, $\mathrm{CA}$ ) and incubated overnight at $4{ }^{\circ} \mathrm{C}$. After extensive washing, the proteins were eluted in $50 \mu \mathrm{l}$ of SDS - PAGE sample buffer and $10 \mu \mathrm{l}$ were electrophoresed on SDS - polyacrylamide gels. The proteins were then transferred to nitrocellulose. For the human sera, Western blot analysis using polyclonal goat anti-MMTV and rabbit anti-goat antibodies was carried out as described in the preceding paragraph. For the control immunoprecipitation performed with the goat anti-MMTV antisera, the Goat TrueBlot Western Blot Kit (eBioscience Inc., San Diego, CA) was used for detection of the viral proteins to prevent obscuring of MMTV bands by the goat heavy and light chain immunoglobulin used in the immunoprecipitation.

\section{RESULTS}

In $65(71 \%)$ of the 92 sera from women with breast cancer, no band was seen in any of the four MMTV immunoblots. One serum demonstrated a smear pattern in three of the four MMTV immunoblots and could not be evaluated. Of the other 26 sera, across all four MMTV immunoblots, 14 (15\%) had one band; two (2\%) had two bands; four (4\%) had three bands; three (3\%) had four bands; two (\#36 and 60) had six bands; and one (\#16) had eight bands (Table 1). Age, breast cancer histology, breast cancer stage, history of cancer treatment, and smoking status did not differ between women whose sera had multiple bands or one band and those who sera had no band (data not presented).

There was heterogeneity in the frequencies of serologic reactivity across the four MMTV strains. Considering all reactivity of all sera (except the one with a smear pattern), there were 19 bands against MMTV strain RIII; 20 bands against strain FM; 18 bands against strain LA; and only five bands against strain $\mathrm{C} 3 \mathrm{H}$. Serum \#60 had two distinct bands of approximate molecular weight 56 and $60 \mathrm{kDa}$ against each strain except $\mathrm{C} 3 \mathrm{H}$, and these differed from the bands that were detected with the $\alpha$-MMTV positive control serum (Figure 1A). Serum \#16 had one or more bands against all four strains, all of which were very faint compared with the $\alpha$-MMTV positive control serum (Figure 1A). None of the bands, including those in sera \#60 and \#16, had the

Table I Prevalence of antibody reactivity against four strains of MMTV among women with breast cancer in the United States

\begin{tabular}{lcc}
\hline Total bands $^{\mathbf{a}}$ & No. (\%) of women & Median (range) age \\
\hline More than 4 & $3(3)$ & $47(37-53)$ \\
4 & $3(3)$ & $48(41-61)$ \\
3 & $4(4)$ & $49(40-58)$ \\
2 & $2(2)$ & $71(55-86)$ \\
1 & $14(15)$ & $53(35-7 I)$ \\
0 & $65(7 \mid)$ & $57(33-95)$ \\
Smear & $1(1)$ & 73 \\
\hline
\end{tabular}

asum of all discrete bands across the immunoblots of all four MMTV strains (RIII, FM, $\mathrm{C} 3 \mathrm{H}$, and $\mathrm{LA}$ ). As shown in Figure IA, serum from Patient \#16 had eight bands (I against RIII, 2 against FM, 2 against $\mathrm{C} 3 \mathrm{H}$, and 3 against $\mathrm{LA}$ ), and sera from Patients \#36 and 60 had six bands (two each of the same molecular weight against RIII, FM, and LA). 

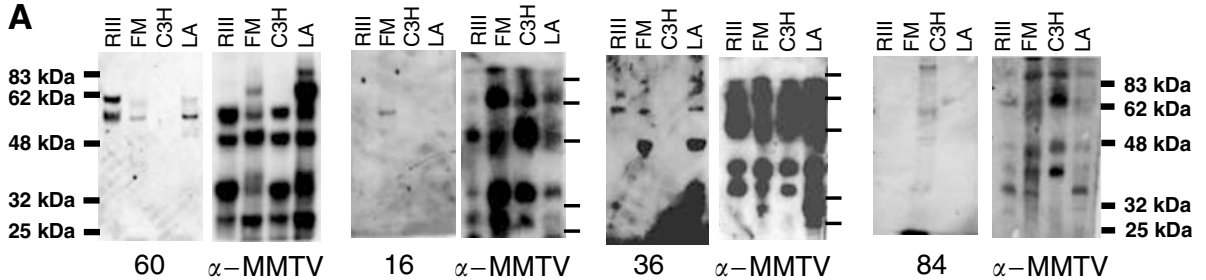

B

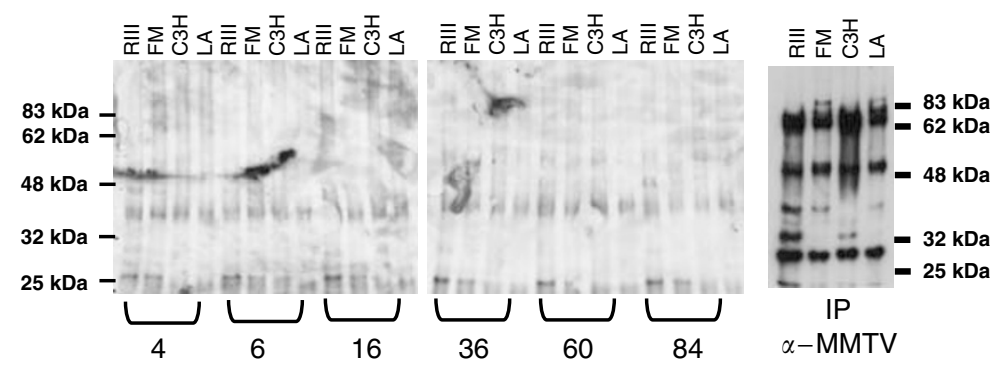

Figure I MMTV antibody immunoblots and immunoprecipitation with human and goat sera. (A) (upper panel). Each human serum was tested by western immunoblot for antibodies against antigens in four strains of MMTV (RIII, FM, C3H, and LA). Four sera (\#60, 16, 36, and 84) demonstrating bands are presented. Each blot was then stripped and re-probed with hyperimmunised goat antiserum (labeled $\alpha$-MMTV). Although various bands are present with each of these selected human sera (e.g., two bands against the RIII. FM, and LA strains in \#36 and 60, four bands against the C3 H strain in \#84), none matched the molecular weight of the bands revealed with the $\alpha$-MMTV positive control serum. (B) (lower panel). Immunoprecipitation of antigens in four strains of MMTV (RIII, FM, C3H, and LA) with six human sera and with hyperimmunised goat antiserum ( $\alpha$-MMTV). MMTV antigens precipitated only with the $\alpha$-MMTV positive control serum.

same molecular weight as the $\alpha$-MMTV positive control serum on the same blot strips (Figure $1 \mathrm{~A}$ ).

In contrast, the masked dilutions of the three positive control reagents performed as expected. The caprine $\alpha$-MMTV and polyclonal $\alpha$-gp52 reagents were detected at the final dilution, 1:10000; further dilutions were not tested. The monoclonal hybridoma supernatant containing $\alpha$-gp52 antibodies was detected at $1: 100$ but not at $1: 1000$ or $1: 10000$. No antibody bands were seen in an unmasked human negative control serum. Rubella antibodies were detected in $85(92 \%)$ of the 92 human sera. Rubella antibodies were not detected in the three neat caprine and monoclonal reagents, but they were detected in the reagents that had been diluted with human plasma-derived Basematrix.

Immunoprecipitation assays were performed with the sera that had four or more bands on immunoblots to determine whether the reactivity was directed against MMTV proteins. As shown in Figure $1 \mathrm{~B}$, these six sera $(\# 4,6,16,36,60$, and 84$)$ had faint background staining with prolonged exposure $(20 \mathrm{~min})$, most likely due to proteins present in the human sera, since they occurred with virus partially purified from different sources (milk (RIII), tumour tissue (FM and LA), and cultured cells $(\mathrm{C} 3 \mathrm{H})$ ). Importantly, the human sera did not immunoprecipitate MMTVspecific antigens from any of the four strains. In contrast, MMTV antigens were readily precipitated with the diluted goat $\alpha$-MMTV positive control serum ( $5 \mathrm{~s}$ exposure). These data indicate that the bands detected by the human serum samples were not MMTV viral proteins.

\section{DISCUSSION}

We found that nearly $30 \%$ of sera from US women with breast cancer had some serologic reactivity against protein in one or more of four different MMTV strains. However, none of this reactivity was specifically directed against MMTV antigens. This reactivity could be due to contaminating cellular or milk proteins present in the partially purified virion preparations. Alternatively, the MMTV envelope, like all retroviruses, incorporates many nonviral, cellular proteins as it is released from the cell. These nonviral proteins can be immunogenic, as illustrated by the protection of macaques against simian immunodeficiency virus (SIV) by vaccination with cells that did not contain SIV (Stott, 1991). Given the similarities across mammalian species, it is not surprising that some women would have developed antibodies against one or more nonviral proteins in the preparations of MMTV that we used. While it is possible that some of the women with reactive sera were exposed to mice, it is more likely that the antibodies we detected were cross-reactive with other cellular antigens, possibly including auto-antibodies (Tax and Manson, 1987; Kovarik et al, 1989). Development of antibodies against nonspecific autoantigens occurs frequently among women with breast cancer (Coronella-Wood and Hersh, 2003).

Mice infected as adults that develop MMTV-induced mammary cancers have life-long, high-titer antibodies against both MMTV and heterophile antigens, (Bowen et al, 1976) whereas mice infected as neonates generally have only a transient antibody response (Luther et al, 1997; Purdy et al, 2003). Seronegative MMTV infection of newborn humans resulting in breast cancer is unlikely, because breast cancer risk is not increased among women who were themselves breast-fed (Titus-Ernstoff et al, 1998). Thus, if MMTV infects humans, antibodies should be detected. Unlike sera from mice infected as adults, none of 91 sera from our women with breast cancer had specific, much less high titer, antibodies against MMTV. The upper 95\% confidence limit for 0/91 implies that MMTV seroprevalence among women with breast cancer does not exceed 3\%.

Antibodies should be present if genes that code for MMTV envelope or other structural proteins are expressed in humans. There are, however, no published reports of anti-MMTV seropositivity among breast cancer patients in whom MMTV RNA expression was found, nor among those in whom DNA sequences resembling MMTV were found (Holland and Pogo, 2004). Likewise, there are no reports of MMTV DNA detection among such patients who were putatively MMTV seropositive. One laboratory, using PCR-based approaches, has reported detection of MMTV-like DNA in liver biopsies and lymph nodes, as well as 
MMTV-like RNA in serum (Mason et al, 2004). Moreover, they found anti-MMTV antibodies in seven of nine patients with primary biliary cirrhosis (Mason et al, 2004). In that study, MMTV antibodies were detected by immunoblot analysis of extracts made from the Mm5MT cell line, the same cells from which we purified the MMTV $(\mathrm{C} 3 \mathrm{H})$ virions, and they used the same polyclonal goat $\alpha$-MMTV antisera for a positive control. However, in a much larger series of primary biliary cirrhosis patients, a different laboratory was unable to detect MMTV-like DNA in either liver biopsies or peripheral blood mononuclear cells by PCR or antibodies against three different strains of MMTV, including the C3H strain (Selmi et al, 2004). The human endogenous retrovirus (HERV) K10 has substantial homology with MMTV, and specific antibodies can be detected when HERV K10 envelope or core (gag) is expressed, as occurs in some cases of testis cancer (Goedert et al, 1999). Mant et al (2004) have suggested that the MMTV-like DNA sequences detected in humans are not MMTV or HERV K10 but rather are another homologous region of the human genome.

Our study has several weaknesses and strengths. The sera that we evaluated were collected $15-20$ years earlier, during which time they might have deteriorated. However, storage of this collection at or below $-70^{\circ} \mathrm{C}$ for up to 10 years was shown to have negligible effect on serum chemistry values other than bilirubin and creatinine (Dimagno et al, 1989). Moreover, some 15 years after they were collected, sera from this collection were proven to contain antibodies against human papilloma virus type 16 and adeno-associated virus (Strickler et al, 1998; Strickler et al, 1999). More directly, in the current study, we found that $92 \%$ of the sera that we tested had rubella antibodies, as expected for women born before 1960. The sera were collected from one large center in the US, were accompanied by limited clinical data, and are not necessarily representative of all US women with breast cancer. To strengthen our study, among the 92 sera from women with breast cancer, we interspersed dilutions of masked positive control sera. These controls demonstrated that our methods could detect MMTV antibodies in masked goat sera and $\alpha$-gp52 monoclonal hybridoma supernatant. To maximise sensitivity for detecting anti-MMTV in human sera, we used four purified MMTV immunoblot preparations, as well as prolonged exposure times. To maximise specificity of the anti-MMTV reactivity, we used immunoprecipitation, as well as overlaying immunoblots probed with caprine $\alpha$-MMTV to directly compare the number, strength, and molecular weights of immunoblot bands found with human sera. In contrast to the $\alpha$-MMTV positive control serum, the human sera were negative or had only weak, nonspecific reactivity.

In summary, we found no evidence of antibodies against MMTV among US women with breast cancer.

\section{ACKNOWLEDGEMENTS}

This work was supported by PHS R01 CA73746 to SRR. Thanks to Ming Shen, Carla Chorley, Janis Koci, and Violet Devairakkam for technical assistance, to the Immunology and Histocompatibility Laboratories of the University of Pennsylvania Department of Pathology and Laboratory Medicine for performing the rubella serology, and to Dr Robert Biggar for review of the manuscript.

\section{REFERENCES}

Bowen JM, Dmochowski L, Miller MF, Priori ES, Seman G, Dodson ML, Maruyama K (1976) Implications of humoral antibody in mice and humans to breast tumor and mouse mammary tumor virus-associated antigens. Cancer Res 36: 759-764

Coronella-Wood JA, Hersh EM (2003) Naturally occurring B-cell responses to breast cancer. Cancer Immunol Immunother 52: 715-738

Dimagno EP, Corle D, O’Brien JF, Masnyk IJ, Go VL, Aamodt R (1989) Effect of long-term freezer storage, thawing, and refreezing on selected constituents of serum. Mayo Clin Proc 64: 1226-1234

Dion AS, Girardi AJ, Williams CC, Pomenti AA, Redfield ES (1987) Responses of serum from breast cancer patients to murine mammary tumor virus: fact or artifact? J Natl Cancer Inst 79: 207-211

Dzuris JL, Zhu W, Kapkov D, Golovkina TV, Ross SR (1999) Expression of mouse mammary tumor virus envelope protein does not prevent superinfection in vivo or in vitro. Virology 263: 418-426

Goedert JJ, Sauter ME, Jacobson LP, Vessella RL, Hilgartner MW, Leitman SF, Fraser MC, Mueller-Lantzsch NG (1999) High prevalence of antibodies against HERV-K10 in patients with testicular cancer but not with AIDS. Cancer Epidemiol Biomarkers Prev 8: 293-296

Holland JF, Pogo BG (2004) Mouse mammary tumor virus-like viral infection and human breast cancer. Clin Cancer Res 10: 5647-5649

Kovarik A, Hlubinova K, Prachar J, Simkovic D, Knotek J (1989) No significant correlation between specific antibodies to mouse mammary tumour virus and human cancer. Br J Cancer 60: $572-575$

Le Bon A, Wache AC, Papiernik M (1999) In vivo elimination of viral superantigen-activated CD4+ T cells: apoptosis occurs at a distance from the activation site. Int Immunol 11: 373-382

Luther SA, Maillard I, Luthi F, Scarpellino L, Diggelmann H, Acha-Orbea H (1997) Early neutralizing antibody response against mouse mammary tumor virus: critical role of viral infection and superantigen-reactive $\mathrm{T}$ cells. J Immunol 159: 2807-2814

Mant C, Gillett C, D’Arrigo C, Cason J (2004) Human murine mammary tumour virus-like agents are genetically distinct from endogenous retroviruses and are not detectable in breast cancer cell lines or biopsies. Virology 318: $393-404$

Mason AL, Farr GH, Xu L, Hubscher SG, Neuberger JM (2004) Pilot studies of single and combination antiretroviral therapy in patients with primary biliary cirrhosis. Am J Gastroenterol 99: 2348-2355

Purdy A, Case L, Duvall M, Overstrom-Coleman M, Monnier N, Chervonsky A, Golovkina T (2003) Unique resistance of I/LnJ mice to a retrovirus is due to sustained interferon gamma-dependent production of virus-neutralizing antibodies. J Exp Med 197: 233-243

Selmi C, Ross SR, Ansari AA, Invernizzi P, Podda M, Coppel RL, Gershwin ME (2004) Lack of immunological or molecular evidence for a role of mouse mammary tumor retrovirus in primary biliary cirrhosis. Gastroenterology 127: 493 - 501

Stott EJ (1991) Anti-cell antibody in macaques. Nature 353: 393

Strickler HD, Schiffman MH, Shah KV, Rabkin CS, Schiller JT, Wacholder S, Clayman B, Viscidi RP (1998) A survey of human papillomavirus 16 antibodies in patients with epithelial cancers. Eur J Cancer Prev 7: 305 - 313

Strickler HD, Viscidi R, Escoffery D, Rattray C, Kotloff KL, Goldberg J, Manns A, Rabkin CS, Daniel R, Hanchard B, Brown C, Hutchinson M, Zanizer D, Palefsky J, Burk R, Cranston B, Clayman B, Shah K (1999) Adeno-associated virus and development of cervical neoplasia. I Med Virol 59: $60-65$

Tax A, Manson LA (1987) A subset of normal human B lymphocytes expresses an antigen cross-reactive with gp52 of murine mammary tumor virus. Cancer Detect Prev Suppl 1: 183-188

Titus-Ernstoff L, Egan KM, Newcomb PA, Baron JA, Stampfer M, Greenberg ER, Cole BF, Ding J, Willett W, Trichopoulos D (1998) Exposure to breast milk in infancy and adult breast cancer risk. J Natl Cancer Inst 90: $921-924$ 